\title{
Population-Based Study of Incidence and Antimicrobial Resistance Patterns of Extraintestinal Escherichia coli Infections in Children in Olmsted County, Minnesota, 2012
}

\author{
Adaora S. Uzodi, MD, MPH${ }^{1}$, Christine M. Lohse, $\mathbf{M S}^{2}$, and Ritu Banerjee, MD, PhD $^{2}$ \\ ${ }^{1}$ Our Lady of the Lake Children's Hospital, Baton Rouge, LA, USA \\ ${ }^{2}$ Mayo Clinic, Rochester, MN, USA
}

\section{Introduction}

Reported cases of antimicrobial-resistant extraintestinal Escherichia coli infections are increasing. ${ }^{1}$ These infections may result in poor outcomes and increased health care costs. ${ }^{2}$ The emergence of antimicrobial resistance in $E$ coli has increased faster than the development of new antibiotics, leading to a paucity of therapeutic agents for highly antimicrobial-resistant strains. Although the increase in antimicrobial-resistant $E$ coli has been well described in adults, the population level incidence of extraintestinal $E$ coli infections in children, including those caused by antimicrobial-resistant strains, is not known. Therefore, we determined the incidence and antimicrobial resistance profiles of extraintestinal $E$ coli infections in children in Olmsted County, Minnesota. Knowledge of the burden of $E$ coli infections, including those caused by multidrug-resistant strains, would support the need for preventive interventions and inform antimicrobial treatment choices.

\section{Methods}

We conducted a population-based study using the Rochester Epidemiology Project (REP), a research infrastructure linking medical records of almost all Olmsted County residents from their primary sources of health care. Olmsted County is located in southeastern Minnesota and had an estimated population of 150287 in $2014 .{ }^{3}$ County residents receive nearly all of their medical care at Mayo Clinic or Olmsted Medical Center. The institutional review boards of Mayo Clinic and Olmsted Medical Center approved this study.

\footnotetext{
Reprints and permissions: sagepub.com/journalsPermissions.nav

Corresponding Author: Ritu Banerjee, Division of Pediatric Infectious Diseases, Department of Pediatrics and Adolescent Medicine, Mayo Clinic, 200 First Street SW, Rochester, MN 55905, USA. banerjee.ritu@ mayo.edu.

The content is solely the responsibility of the authors and does not necessarily represent the official views of the National Institutes of Health.

Author Contributions

ASU designed the data collection instruments, performed data abstraction, drafted and revised the manuscript, and approved the final manuscript as submitted. CML performed statistical analyses, reviewed and revised the manuscript, and approved the final manuscript as submitted. RB designed the study, reviewed and revised the manuscript, and approved the final manuscript as submitted.

Declaration of Conflicting Interests

The author(s) declared no potential conflicts of interest with respect to the research, authorship, and/or publication of this article.
} 


\section{Study Population}

Patients were identified by querying the clinical microbiology laboratory databases of Mayo Clinic and Olmsted Medical Center for all cultures with growth of $E$ coli from extraintestinal sources (blood, sterile body fluids, urine, respiratory, wound) from children 0 to 18 years old, obtained from January 1, 2012 through December 31, 2012. We included only children who were residents of Olmsted County and had documented Minnesota state research authorization (Minnesota Statute, section 144.295). We excluded children without state research authorization or whose $E$ coli isolates lacked antimicrobial susceptibility results. If a patient had multiple positive cultures during the study period, the first isolate was included, or sterile sources were prioritized over nonsterile or urine sources (ie, blood was included rather than urine). Polymicrobial cultures with growth of $E$ coli from any extraintestinal source except urine were included as incident cases. Urine cultures with monomicrobial $E$ coli growth at $>10^{5} \mathrm{CFU} / \mathrm{mL}$ were included.

\section{Definitions}

An isolate was classified as multidrug-resistant (MDR) if it was resistant to 3 or more of the following 8 antimicrobial drugs/drug classes: (1) ampicillin/sulbactam, (2) piperacillin/ tazobactam, (3) trimethoprim/sulfamethoxazole (TMP/SMX), (4) fluoroquinolones (ciprofloxacin or levofloxacin), (5) aminoglycosides (gentamicin, tobramycin, or amikacin), (6) first- or second-generation cephalosporins (cefazolin, cephalothin, or cefuroxime), (7) extended-spectrum cephalosporins (ceftriaxone, cefotaxime, ceftazidime or cefepime), and (8) carbapenems (meropenem or ertapenem). ${ }^{4}$ Pan-susceptible isolates were sensitive to all of the antimicrobials listed above.

We distinguished infection from colonization among isolates from respiratory, urine, or wound specimens as follows: A urinary $E$ coli isolate represented a urinary tract infection (UTI) when the patient exhibited all of the following: fever, abdominal pain or dysuria, pyuria (defined as $>5$ white blood cells/high-power field or positive leukocyte esterase), and monomicrobial growth of E coli at $>10^{5} \mathrm{CFU} / \mathrm{mL}$. Lower respiratory tract specimens represented infection (pneumonia, bronchitis, or tracheitis) when patients had the following: fever or cough or respiratory distress and supportive objective findings (infiltrates on chest imaging or signs of airway inflammation on bronchoscopy). E coli from wound cultures represented wound infections when patients had accompanying signs of ongoing inflammation from the culture site such as pain, erythema, discharge, or fever. Patients who had urine, respiratory, and wound $E$ coli isolates but did not fulfill the above criteria for infection were considered colonized and excluded from incidence estimates.

\section{Statistical Analysis}

Incidence rates were calculated using the incident cases (defined above) as the numerator and age- and sex-specific counts of the Olmsted County population as the denominator. The denominator was obtained from complete enumeration of the Olmsted County population provided by the REP. ${ }^{5}$ Because the population of Olmsted County is predominantly white, incidence rates were directly age- and sex-adjusted to the structure of the 2010 US white population. The relationships of age group and sex with incidence rates were assessed by fitting Poisson regression models using the SAS procedure GENMOD. Statistical analyses 
were performed using the SAS software package (SAS Institute, Cary, NC). All tests were 2sided and $P<.05$ was considered statistically significant.

\section{Results}

\section{Incidence of $E$ coli Infection Among Children in Olmsted County, Minnesota, 2012}

During the study period, there were 217 distinct children with $E$ coli infection. The median age was 7 years and 199 (92\%) were female. The overall incidence of $E$ coli infection was 535 per 100000 person-years. Incidence differed significantly by age group $(P<.001)$ and gender $(P<.001)$ (Figure 1A). Among males, a sustained decline in the incidence rate was noted with increasing age. In contrast, among females, incidence peaked in infancy, decreased through school age, and then rose dramatically in the 16- to 18-year-old age group. UTIs accounted for the vast majority of extraintestinal $E$ coli infections (97\%); other sites of infection were far less common (bloodstream, 1.4\%; respiratory tract, abdominal and soft tissue infections combined 1.8\%). The age- and sex-adjusted incidence per 100000 person-years was 519 for $E$ coli UTI and 6 for $E$ coli bloodstream infection.

Among the 217 children with $E$ coli infections, 22 (10\%) had MDR isolates, 119 (55\%) had pan-susceptible isolates, and $76(35 \%)$ had isolates that were resistant to 1 to 2 drug classes. Twenty-one of $22(95 \%)$ MDR infections were UTIs and 1 was a respiratory tract infection. The incidence per 100000 person-years of MDR E coli infection was 53 overall, 89 among females, and 19 among males.

\section{Antimicrobial Resistance Rates}

Antimicrobial resistance rates of the $E$ coli isolates are shown in Figure 1B. Resistance to extended-spectrum penicillins, third- and fourth-generation cephalosporins, carbapenems, nitrofurantoin, fluoroquinolones, and aminoglycosides was low ( $0 \%$ to $4 \%$ ). In contrast, resistance rates were higher for ampicillin (43\%), ampicillin-sulbactam (55\%), trimethoprim-sulfamethoxazole (20\%), and first-generation cephalosporins (15\% to 24\%).

\section{Discussion}

This is the first study to provide population-level incidence rates of all extraintestinal $E$ coli infections in a contemporary cohort of US children. We found that the incidence per 100000 person-years was 535 for all pediatric $E$ coli infections, 519 for $E$ coli UTI, and 6 for $E$ coli bloodstream infections. These incidence rates are slightly higher than those reported in previous studies from our region of specific infectious syndromes caused by $E$ coli. Among children in our county, incidence per 100000 person- years was 413 for $E$ coli bacteriuria in $2009,{ }^{6}$ and 3.5 for $E$ coli bloodstream infection in $2007 .{ }^{7}$ These data demonstrate that among children, the burden of E coli infections is high, and comparable to that of other important pathogens. For example, the incidence of Staphylococcus aureus bloodstream infections among children in our county between 2003 and 2005 was 6.1 per $100000 .^{8}$

We observed low resistance rates among pediatric $E$ coli isolates to extended-spectrum penicillins, third- and fourth-generation cephalosporins, carbapenems, quinolones, and aminoglycosides. We also noted a relatively low rate of MDR infections although there are 
no local or national data for comparison. Resistance to trimethoprim-sulfamethoxazole was higher (20\%), but similar to results from national data. ${ }^{9}$ However, we noted higher prevalence of resistance to ampicillin-sulbactam (55\%), first-generation cephalosporins cefazolin (15\%), and cephalothin (24\%) compared with results from a 2009 national survey of pediatric outpatient $E$ coli UTIs ${ }^{9}$ and a previous study of $E$ coli bloodstream infections in our county from 1998 to $2007 .{ }^{10}$ Thus, extraintestinal pediatric $E$ coli isolates in our area have become increasingly resistant to first-generation cephalosporins, perhaps due to more frequent use of these agents for empiric management of UTI.

The resistance rates observed in our population are similar to but slightly lower than the pooled prevalence of resistance reported from other developed countries in a recent global study of pediatric $E$ coli urinary tract infections. ${ }^{1}$ In this systematic review, the highest resistance rates were seen with ampicillin (53\%) and trimethoprim- sulfamethoxazole (24\%), while resistance rates to third generation cephalosporins, ciprofloxacin, and nitrofurantoin were less than $2.5 \%$, similar to our population.

Our study has limitations. We were unable to study trends in incidence as we collected data only over 1 year. We collected data retrospectively, which may have led to selection and misclassification biases. Our study was conducted in a single geographic region and findings may not be generalizable to other communities or institutions with different patient populations and antibiotic resistance phenotypes. Despite these limitations, study strengths are that we used stringent criteria for defining a clinically significant culture, and were able to compare our results to prior population-based estimates from our county.

In conclusion, in our region of the US Midwest, the incidence of extraintestinal pediatric $E$ coli UTI and bloodstream infection in 2012 is higher than observed in prior years. Furthermore, E coli isolates demonstrate increased resistance to first generation cephalosporins, commonly used first-line agents for treatment of $E$ coli UTI. Providers should be aware of the changing epidemiology of $E$ coli infections in children.

\section{Acknowledgments}

We thank the staff of the Rochester Epidemiology Project and the clinical microbiology laboratories of Mayo Clinic and Olmsted Medical Center for providing us with microbiology data. We are also grateful to Louis Schencke and Ann Houtsma for their assistance with database design and chart abstraction and Drs Larry Baddour, Thomas Boyce, and W. Charles Huskins for their guidance.

\section{Funding}

The author(s) disclosed receipt of the following financial support for the research, authorship, and/or publication of this article: This work was supported in part by the Department of Pediatric and Adolescent Medicine, Mayo Clinic, Rochester, Minnesota, the Mayo Clinic Center for Clinical and Translational Science grant (UL1 TR000135) and the National Institute on Aging of the National Institutes of Health (Award Number R01AG034676).

\section{References}

1. Bryce A, Alastair DH, Lane IF, Thornton HV, Wootton M, Costelloe C. Global prevalence of antibiotic resistance in paediatric urinary tract infections caused by Escherichia coli and association with routine use of antibiotics in primary care: systematic review and meta-analysis. BMJ. 2016; 352:i1939. 
2. Centers for Disease Control and Prevention. [Accessed June 10, 2016] Antibiotic resistance threats in the united states. 2013. http://www.cdc.gov/drugresistance/threat-report-2013/

3. US Census Bureau. [Accessed June 10, 2016] http://quickfacts.census.gov/qfd/states/27/27109.html

4. Magiorakos AP, Srinivasan A, Carey RB, et al. Multidrug-resistant, extensively drug-resistant and pandrug-resistant bacteria: an international expert proposal for interim standard definitions for acquired resistance. Clin Microbiol Infect. 2012; 18:268-281. [PubMed: 21793988]

5. St Sauver JL, Grossardt BR, Yawn BP, Melton LJ 3rd, Rocca WA. Use of a medical records linkage system to enumerate a dynamic population over time: the Rochester Epidemiology Project. Am J Epidemiol. 2011; 173:1059-1068. [PubMed: 21430193]

6. Swami SK, Liesinger JT, Shah N, Baddour LM, Banerjee R. Incidence of antibiotic-resistant Escherichia coli bacteriuria according to age and location of onset: a population-based study from Olmsted County, Minnesota. Mayo Clin Proc. 2012; 87:753-759. [PubMed: 22795635]

7. Al-Hasan MN, Huskins WC, Lahr BD, Eckel-Passow JE, Baddour LM. Epidemiology and outcome of Gram-negative bloodstream infection in children: a population-based study. Epidemiol Infect. 2011; 139:791-796. [PubMed: 20598212]

8. Uslan DZ, Crane SJ, Steckelberg JM, et al. Age- and sex-associated trends in bloodstream infection: a population-based study in Olmsted County, Minnesota. Arch Intern Med. 2007; 167:834-839. [PubMed: 17452548]

9. Edlin RS, Shapiro DJ, Hersh AL, Copp HL. Antibiotic resistance patterns of outpatient pediatric urinary tract infections. J Urol. 2013; 190:222-227. [PubMed: 23369720]

10. Al-Hasan MN, Lahr BD, Eckel-Passow JE, Baddour LM. Antimicrobial resistance trends of Escherichia coli bloodstream isolates: a population-based study, 1998-2007. J Antimicrob Chemother. 2009; 64:169-174. [PubMed: 19435736] 


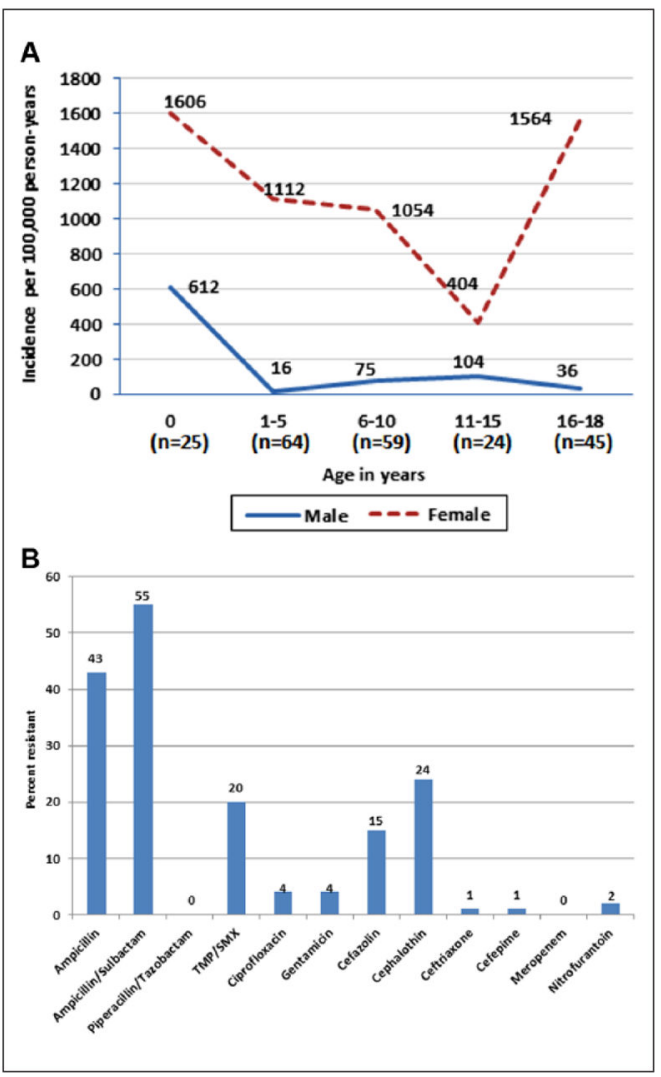

Figure 1.

(A) Incidence of Escherichia coli infection by age and gender among pediatric residents of Olmsted Country, Minnesota in 2012. (B) Antimicrobial resistance among 217 pediatric $E$ coli isolates in Olmsted Country, Minnesota in 2012. TMP/SMX, trimethoprim-

sulfamethoxazole. Susceptibility testing done for a subset of 217 isolates for ampicilin/ sulbactam $(n=134)$, cephalothin $(n=134)$, ceftriaxone $(n=144)$, cefeprime $(n=142)$, and nitrofurantoin $(\mathrm{n}=211)$. 